\title{
OPEN Gestational diabetes mellitus and the role of intercurrent type 2 diabetes on long-term risk of cardiovascular events
}

\author{
Jiyu Sun ${ }^{1,5}$, Gyu Ri Kim²,3,5, Su Jin Lee ${ }^{4 \bowtie}$ \& Hyeon Chang Kim ${ }^{2 \bowtie}$
}

Recent studies have shown that gestational diabetes mellitus (GDM) is associated with an increased risk for cardiovascular disease. GDM has also been shown to be a risk factor for type 2 diabetes (T2DM) after pregnancy. However, there is limited evidence regarding the role of intercurrent T2DM on the relationship between GDM and future CVD. Thus, we investigated the risks of incident cardiovascular events among women with GDM during pregnancy compared to women without GDM and whether the increased CVD risk is dependent on intercurrent development of T2DM. We conducted a population-based retrospective cohort study using the Korean National Health Insurance Service claims database. Outcomes were the first occurrence of any CVD (myocardial infarction, treatment with coronary revascularization, heart failure, and cerebrovascular disease). Cox proportional hazard models were used to assess the association between GDM and incident CVD events, using landmark analysis at 4 years. A total of 1,500,168 parous women were included in the analysis, of which 159,066 $(10.60 \%)$ had GDM. At a median follow-up of 12.8 years, 13,222 incident cases of total CVD were observed. Multivariable-adjusted hazard ratio for total CVD among women with prior GDM, compared with those without GDM, was 1.08 (95\% Cl 1.02-1.14). Further classifying GDM by progression to T2DM in relation to total CVD risk indicated a positive association for GDM with progression to T2DM vs no GDM or T2DM (HR 1.74; 95\% Cl 1.40-2.15), and no statistically significant association for GDM only (HR 1.06; 95\% Cl 1.00-1.12). GDM with subsequent progression to T2DM were linked with an increased risk of cardiovascular diseases. These findings highlight the need for more vigilant postpartum screening for diabetes and the implementation of diabetes interventions in women with a history of GDM to reduce future CVD risk.

Epidemiological studies have established that gestational diabetes mellitus (GDM), characterized by transitory form of diabetes induced by glucose intolerance and pancreatic $\beta$-cell dysfunction with onset or first recognition during pregnancy, is associated with cardiovascular risk factors such as type 2 diabetes (T2DM) ${ }^{1-3}$. In a meta-analysis of 20 studies with more than 670,000 participants, GDM has been shown to confer more than sevenfold increased risk of developing $\mathrm{T}_{2} \mathrm{DM}^{4}$. However, there is limited and inconclusive evidence for the effects of GDM on long-term risk of cardiovascular disease (CVD) and it is uncertain whether such association is dependent upon intercurrent progression to $\mathrm{T}_{2} \mathrm{DM}^{5-8}$. National health registry data from Sweden, showed that women with GDM have a considerable risk of developing CVD, even after adjustment for subsequent T2DM ${ }^{3}$. Yet, other studies have found that controlling for T2DM fully attenuates the increased risk of CVD in women with diagnosis of GDM in pregnancy ${ }^{6,7}$.

With the absolute number of deaths attributable to CVD rising steadily from 12.31 million in 1990 reaching 18.6 million in the year 2019, CVD is the leading global cause of mortality and disability-adjusted life years ${ }^{9}$. Although the CVD mortality rates are much lower among women compared with men, CVDs, principally coronary heart disease and stroke, are responsible for over 8.6 million deaths among women per year ${ }^{10,11}$. Given the increasing global burden of cardiovascular diseases among women, it is of great public health significance to identify factors that contribute to worsening CVD risk profiles. Accordingly, we sought to investigate the

\footnotetext{
${ }^{1}$ Medical Research Collaborating Center, SMG-SNU Boramae Medical Center, Seoul, Korea. ${ }^{2}$ Department of Preventive Medicine, Yonsei University College of Medicine, Seoul, Korea. ${ }^{3}$ Institute of Health Services Research, Yonsei University, Seoul, Korea. ${ }^{4}$ Department of Internal Medicine, Seoul Red Cross Hospital, Seoul, Korea. ${ }^{5}$ These authors contributed equally: Jiyu Sun and Gyu Ri Kim.『email: kibalhae82@gmail.com; hckim@yuhs.ac
} 


\begin{tabular}{|c|c|c|c|c|c|c|c|}
\hline & \multicolumn{6}{|c|}{ GDM diagnosis } & \multirow[b]{3}{*}{ P-value ${ }^{\dagger}$} \\
\hline & \multicolumn{2}{|l|}{\begin{tabular}{|l|} 
Total \\
\end{tabular}} & \multicolumn{2}{|l|}{ No } & \multicolumn{2}{|l|}{ Yes } & \\
\hline & $\mathbf{N}$ & $\%$ & $\mathbf{N}$ & $\%$ & $\mathrm{~N}$ & $\%$ & \\
\hline Number & $1,500,168$ & 100.00 & $1,341,102$ & 89.40 & 159,066 & 10.60 & \\
\hline Age at index date, years & & & & & & & $<0.0001$ \\
\hline $20-24$ & 119,566 & 7.97 & 111,559 & 8.32 & 8007 & 5.03 & \\
\hline $25-29$ & 624,771 & 41.65 & 560,366 & 41.78 & 64,405 & 40.49 & \\
\hline $30-34$ & 589,440 & 39.29 & 523,347 & 39.02 & 66,093 & 41.55 & \\
\hline $35-39$ & 149,382 & 9.96 & 131,082 & 9.77 & 18,300 & 11.51 & \\
\hline$\geq 40$ & 17,009 & 1.13 & 14,748 & 1.11 & 2261 & 1.42 & \\
\hline Socioeconomic status & & & & & & & $<0.0001$ \\
\hline Low income & 335,050 & 22.84 & 299,590 & 22.86 & 35,460 & 22.73 & \\
\hline Middle-low & 423,376 & 28.86 & 379,103 & 28.92 & 44,273 & 28.38 & \\
\hline Middle-high & 471,121 & 32.13 & 420,816 & 32.10 & 50,305 & 32.25 & \\
\hline High income & 237,242 & 16.17 & 211,291 & 16.12 & 25,951 & 16.64 & \\
\hline Total parity & & & & & & & 0.2524 \\
\hline 1 & $1,283,451$ & 85.55 & $1,147,395$ & 85.56 & 136,056 & 85.53 & \\
\hline 2 & 214,411 & 14.29 & 191,670 & 14.29 & 22,741 & 14.30 & \\
\hline$\geq 3$ & 2,306 & 0.15 & 2,037 & 0.15 & 269 & 0.17 & \\
\hline Polycystic ovary syndrome & & & & & & & $<0.0001$ \\
\hline No & $1,489,528$ & 99.29 & $1,332,154$ & 99.33 & 157,374 & 98.94 & \\
\hline Yes & 10,640 & 0.71 & 8948 & 0.67 & 1692 & 1.06 & \\
\hline Preeclampsia or hypertension & & & & & & & $<0.0001$ \\
\hline No & $1,427,271$ & 95.14 & $1,279,883$ & 95.44 & 147,388 & 92.66 & \\
\hline Yes & 72,897 & 4.86 & 61,219 & 4.56 & 11,678 & 7.34 & \\
\hline Dyslipidemia & & & & & & & $<0.0001$ \\
\hline No & $1,381,579$ & 92.09 & $1,240,853$ & 92.52 & 140,726 & 88.47 & \\
\hline Yes & 118,589 & 7.91 & 100,249 & 7.48 & 18,340 & 11.53 & \\
\hline
\end{tabular}

Table 1. General characteristics of the study population at index date according to gestational diabetes mellitus diagnosis. GDM Gestational diabetes mellitus. ${ }^{\dagger} p$-values from the Chi-square test.

relationship between GDM and the risk of cardiovascular events using the Korean National Health Insurance Service (NHIS) database and examine the role of intercurrent T2DM in determining CVD outcomes in women with previous GDM.

\section{Results}

Participant characteristics. 1,500,168 parous women aged 20-49 years were available for analysis, of which 159,066 (10.6\%) reported having GDM while 1,341,102 (89.4\%) women did not have GDM. General characteristics of the study population at index date according to GDM status are summarized in Table 1. Compared with non-GDM women, those with GDM were generally more likely to be older and were more often in the higher income groups. Women with GDM were also more likely to have a history of preeclampsia or hypertension, polycystic ovary syndrome, as well as history of dyslipidemia than individuals without GDM. However, no statistically significant difference in parity number was detectable between the two groups.

Over a median follow-up of 12.8 years, there were 13,222 incident CVD cases (including 785 MIs, 298 coronary revascularization, 2705 heart failure, and 10,015 cerebrovascular disease), yielding an incidence rate of 68.95 per 100,000 person-years. The incidence rate of total CVD per 100,000 person-years was 76.78 among women with GDM compared with 68.06 in the reference group of parous women without GDM. Table 2 shows the association between GDM and incident CVD events. In the 4-year landmark analysis, GDM was a significant risk factor for subsequent CVD after controlling for age. Those with GDM experienced approximately $15 \%$ greater risk of CVD during follow-up (HR 1.15; 95\% CI 1.09-1.22). The positive relationship between GDM and risk of CVD was attenuated but remained present after further adjustment for total parity, household income and history of preeclampsia or hypertension, polycystic ovary syndrome, and dyslipidemia (multivariable adjusted HR 1.08; 95\% CI 1.02-1.14). As compared with women without GDM, age-adjusted HRs were significantly elevated in GDM group for each CVD subtype, except for myocardial infarction. The strongest associations with GDM were seen for incident coronary revascularization, followed by heart failure, and cerebrovascular disease. After adjusting for potential confounders, the presence of GDM was independently associated with a 58\% (multivariable adjusted HR 1.58, 95\% CI 1.16-2.14) and 20\% (multivariable adjusted HR 1.20, 95\% CI 1.07-1.35) increased risk of incident coronary revascularization and heart failure, respectively. Yet the positive associations of myocardial infarction and cerebrovascular disease with GMD were found to be non-significant.

Table 3 presents the hazard ratios for incident CVD associated with GDM, accounting for the intercurrent occurrence of T2DM. Characteristics of participants stratified into four groups (No GDM or T2DM, GDM only, 


\begin{tabular}{|c|c|c|c|c|c|c|c|c|c|c|}
\hline \multirow[b]{2}{*}{ Outcomes } & \multicolumn{3}{|c|}{ No GDM $(\mathrm{N}=1,341,102)$} & \multicolumn{3}{|c|}{ GDM $(N=159,066)$} & \multirow{2}{*}{$\begin{array}{l}\text { Age adjusted } \\
\text { HR }(95 \% \mathrm{CI})\end{array}$} & \multirow[b]{2}{*}{ P-value } & \multirow{2}{*}{$\begin{array}{l}\text { Multivariable } \\
\text { HR }(95 \% \text { CI })^{b}\end{array}$} & \multirow[b]{2}{*}{ P-value } \\
\hline & No. of events $\dagger$ & Person-years & Incidence rate $^{a}$ & No. of events $\dagger$ & Person-years & Incidence rate $^{a}$ & & & & \\
\hline Total CVD & 11,705 & $17,199,273$ & 68.06 & 1517 & $1,975,840$ & 76.78 & $1.15(1.09-1.22)$ & $<0.0001$ & $1.08(1.02-1.14)$ & 0.008 \\
\hline $\begin{array}{l}\text { Myocardial } \\
\text { infarction }\end{array}$ & 693 & $17,233,934$ & 4.02 & 92 & $1,980,253$ & 4.65 & $1.20(0.96-1.49)$ & 0.107 & $1.12(0.90-1.40)$ & 0.314 \\
\hline $\begin{array}{l}\text { Coronary revas- } \\
\text { cularization }\end{array}$ & 246 & $17,235,559$ & 1.43 & 52 & $1,980,345$ & 2.63 & $1.80(1.34-2.44)$ & 0.001 & $1.58(1.16-2.14)$ & 0.004 \\
\hline Heart failure & 2367 & $17,230,905$ & 13.74 & 338 & $1,979,655$ & 17.07 & $1.32(1.18-1.48)$ & $<0.0001$ & $1.20(1.07-1.35)$ & 0.002 \\
\hline $\begin{array}{l}\text { Cerebrovascular } \\
\text { disease }\end{array}$ & 8890 & $17,206,520$ & 51.67 & 1125 & $1,976,892$ & 56.91 & $1.11(1.05-1.19)$ & 0.001 & $1.04(0.98-1.11)$ & 0.184 \\
\hline
\end{tabular}

Table 2. Results of landmark analysis for the association between gestational diabetes mellitus and risk of CVD. CVD cardiovascular disease, $H R$ hazard ratio, $C I$ confidence interval. ${ }^{\dagger}$ Note that the number of events in CVD subtypes does not add up to the total CVD cases as the subtypes are not mutually exclusive. ${ }^{\text {ancidence }}$ rate per 100,000 person-years. ${ }^{b}$ Adjusted for age, parity, household income, history of preeclampsia or hypertension, polycystic ovary syndrome, and dyslipidemia.

\begin{tabular}{|c|c|c|c|c|}
\hline Categories & Events $(\mathrm{N})$ & Incidence rate $\mathrm{a}^{\mathrm{a}}$ & Age adjusted HR (95\% CI) & Multivariable HR $(95 \% \mathrm{CI})^{\mathrm{b}}$ \\
\hline \multicolumn{5}{|l|}{ Total CVD } \\
\hline No GDM or T2DM & 11,590 & 67.54 & 1.00 & 1.00 \\
\hline GDM only & 1428 & 73.67 & $1.12(1.06-1.18)$ & $1.06(1.00-1.12)$ \\
\hline T2DM only & 115 & 295.09 & $3.64(3.03-4.38)$ & $2.01(1.66-2.43)$ \\
\hline GDM with progression to T2DM & 89 & 237.18 & $3.09(2.51-3.82)$ & $1.74(1.40-2.15)$ \\
\hline \multicolumn{5}{|l|}{ Myocardial infarction } \\
\hline No GDM or T2DM & 686 & 3.99 & 1.00 & 1.00 \\
\hline GDM only & 85 & 4.38 & $1.14(0.91-1.43)$ & $1.08(0.86-1.37)$ \\
\hline T2DM only & 7 & 17.80 & $3.73(1.77-7.86)$ & $1.79(0.79-4.05)$ \\
\hline GDM with progression to T2DM & 7 & 18.52 & $4.14(1.96-8.73)$ & $2.29(1.07-4.88)$ \\
\hline \multicolumn{5}{|l|}{ Coronary revascularization } \\
\hline No GDM or T2DM & 232 & 1.35 & 1.00 & 1.00 \\
\hline GDM only & 44 & 2.27 & $1.66(1.20-2.29)$ & $1.53(1.10-2.12)$ \\
\hline T2DM only & 14 & 35.61 & $19.11(11.08-32.96)$ & $8.97(5.03-16.00)$ \\
\hline GDM with progression to T2DM & 8 & 21.16 & $11.98(5.90-24.32)$ & $5.51(2.64-11.51)$ \\
\hline \multicolumn{5}{|l|}{ Heart failure } \\
\hline No GDM or T2DM & 2333 & 13.57 & 1.00 & 1.00 \\
\hline GDM only & 310 & 15.96 & $1.25(1.11-1.41)$ & $1.16(1.03-1.31)$ \\
\hline T2DM only & 34 & 86.56 & $5.31(3.78-7.46)$ & $2.61(1.84-3.71)$ \\
\hline GDM with progression to T2DM & 28 & 74.17 & $4.97(3.42-7.22)$ & $2.48(1.70-3.63)$ \\
\hline \multicolumn{5}{|l|}{ Cerebrovascular disease } \\
\hline No GDM or T2DM & 8810 & 51.32 & 1.00 & 1.00 \\
\hline GDM only & 1065 & 54.92 & $1.09(1.02-1.16)$ & $1.03(0.97-1.10)$ \\
\hline T2DM only & 80 & 204.71 & $3.29(2.64-4.09)$ & $1.85(1.46-2.33)$ \\
\hline GDM with progression to T2DM & 60 & 159.63 & $2.69(2.09-3.48)$ & $1.55(1.20-2.02)$ \\
\hline
\end{tabular}

Table 3. Risk of incident CVD associated with GDM and progression to type 2 diabetes. CVD cardiovascular disease, $H R$ hazard ratio, CI confidence interval, GDM gestational diabetes mellitus, T2DM type 2 diabetes

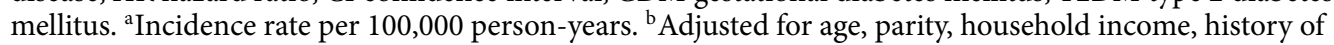
preeclampsia or hypertension, polycystic ovary syndrome, and dyslipidemia.

T2DM only, GDM with progression to T2DM) are shown in Supplementary Table 1. Relative to the reference group of parous women with neither GDM nor T2DM, the corresponding age adjusted HRs for total CVD were 1.12 (95\% CI 1.06-1.18) for women with GDM only, 3.09 (95\% CI 2.51-3.82) and 3.64 (95\% CI 3.03-4.38) for GDM with progression to T2DM and T2DM only, respectively. After further adjustment for relevant covariates, the HRs for total CVD were attenuated (GDM with progression to T2DM: HR 1.74, 95\% CI 1.40-2.15; T2DM only group: HR 2.01, 95\% CI 1.66-2.43), and there was no longer a statistically significant relationship between incident total CVD and GDM only (multivariable adjusted HR 1.06; 95\% CI 1.00-1.12). Analyses of CVD subtypes indicated that the same is true of myocardial infarction and cerebrovascular disease. For example, GDM with subsequent progression to T2DM were linked with over two-fold increased risk of myocardial infarction and 1.55 times greater risk of cerebrovascular disease. However, for women with GDM in whom T2DM did not 

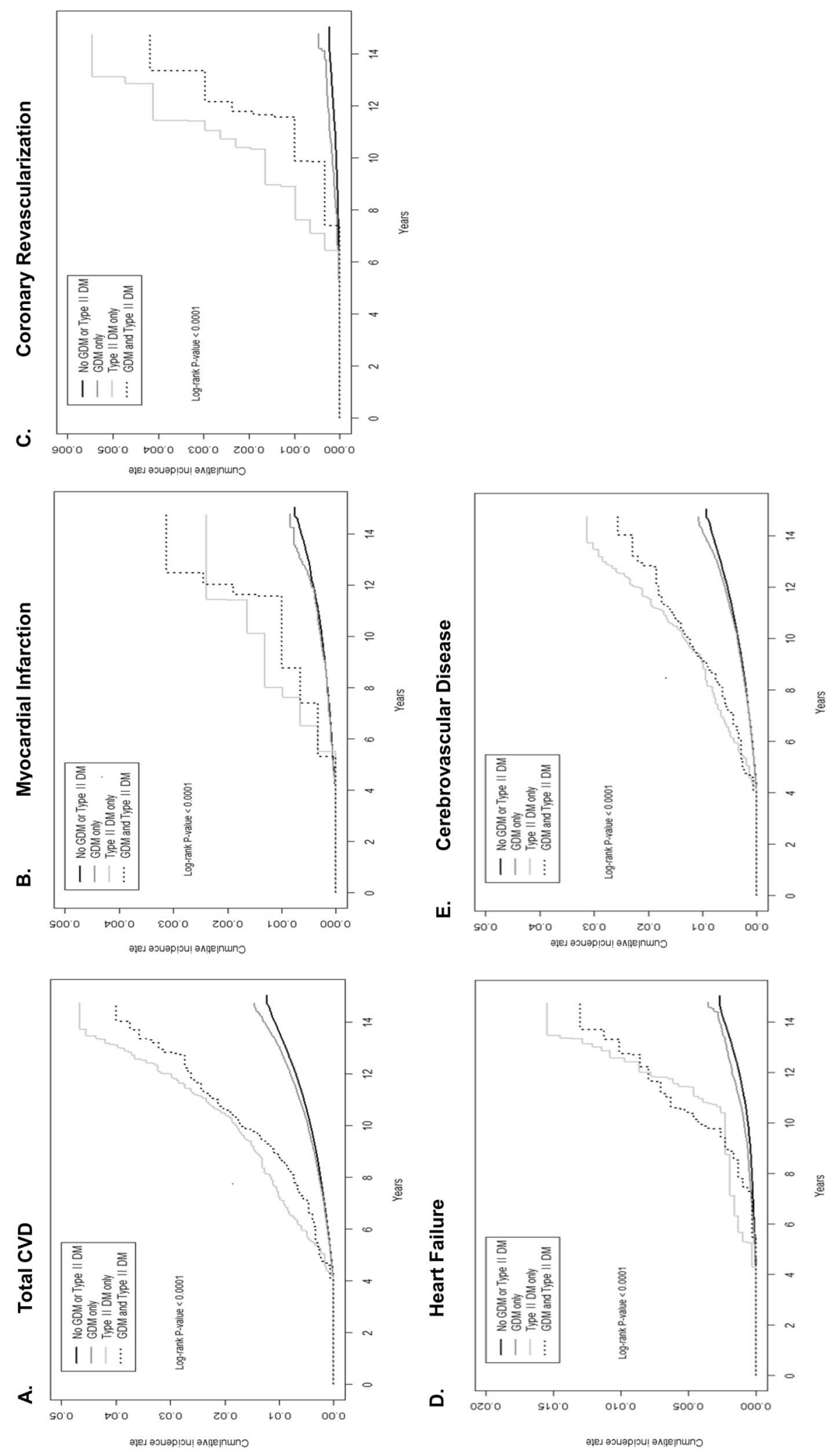

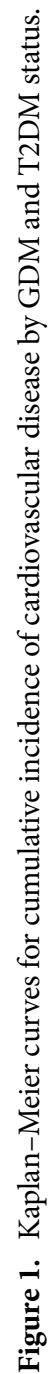


develop, the HRs for myocardial infarction and cerebrovascular disease were 1.08 (95\% CI 0.86-1.37) and 1.03 (95\% CI $0.97-1.10)$, respectively. On the contrary, GDM was significantly and positively associated with coronary revascularization and heart failure, regardless of intercurrent development of T2DM.

The post hoc power calculation indicated that the study had adequate statistical power to detect a clinically significant HR. With our sample size of $1,500,158$, we had $78 \%$ power with a $5 \%$ significance level to detect a HR of 1.08 for total CVD outcome for GDM group vs non-GDM group ${ }^{12,13}$.

Figure 1 shows the Kaplan-Meier plot for the cumulative proportion of patients experiencing total CVD, including results of the log-rank test to compare incidence of CVD events between the exposure groups. The logrank test revealed a statistically significant difference in the incidence of total CVD over time between the four exposure groups $(\mathrm{P}<0.0001)$.

Sensitivity analyses. In order to determine the sensitivity of the findings to the choice of landmark, we conducted a sensitivity analysis using an alternative landmark point at 2 years. As shown in Supplementary Table 2, the results of the sensitivity analysis were not materially different from the results of the main analysis, with a multivariable adjusted HRs for total CVD of 2.24 (95\% CI 1.73-2.92), 2.09 (95\% CI 1.59-2.75), and 1.11 (95\% CI 1.04-1.18) in GDM with progression to T2DM, T2DM only, and GDM only groups, respectively. We also performed a sensitivity analysis of the primary analysis whereby all-cause deaths are included as endpoint events along with CVD. Participants who were still alive or without CVD by the end of the follow-up were treated as censored. There were no meaningful changes in the respective hazard ratios for the GDM with progression to T2DM (HR 1.66; 95\% CI 1.37-2.01), T2DM only (HR 1.97; 95\% CI 1.66-2.33), and GDM only groups (HR 1.04; 95\% CI 0.99-1.09), highlighting the robustness of the primary results (Supplementary Table 3).

\section{Discussion}

This large population-based retrospective study of Korean women demonstrated increased risk of cardiovascular events among individuals diagnosed with glucose intolerance during pregnancy. When examining CVD subtypes, we found that GDM is independently associated with a $58 \%$ and $20 \%$ increased risk of incident coronary revascularization and heart failure, respectively. More importantly, further analysis revealed that subsequent development of T2DM accounts for much of this elevated risk, which reinforces the importance of diabetes prevention strategies in this high-risk population.

Our estimates of the associations between GDM and future CVD are generally in accordance with those of previous studies. Based on a retrospective cohort study, Shah et al. ${ }^{6}$ found that the risk of incident CVD was significantly higher in women with previous GDM relative to age-matched controls. In their study, a hazard ratio of 1.71 (95\% CI 1.08-2.69) was noted for CVD among women with GDM. Similar to our findings, the hazard ratio declined to 1.13 (95\% CI 0.67-1.89) and lost its significance after further controlling for subsequent T2DM. Furthermore, in a meta-analysis of data pooled from 9 studies involving over five million participants, there was nearly twofold excess risk of cardiovascular events in women with history of GDM compared with non-GDM women (95\% CI $1.57-2.50)^{14}$.

Several factors may underlie the observed relationship between GDM and cardiovascular outcomes. Among other potential mechanisms, GDM has shown to be related to diastolic dysfunction and a higher risk of adverse cardiac remodeling patterns, all of which are key factors involved in the development of heart failure ${ }^{15,16}$. Moreover, women with glucose intolerance during pregnancy were observed to exhibit higher left ventricular mass and wall thickness than people who are normoglycemic ${ }^{17}$, possibly explained by the enhanced formation of advanced glycation end products in the myocardium, which may result in endothelium damage and impaired arterial elasticity ${ }^{18}$. Previous research has reported significant abnormalities of cardiovascular system among 31 women with GDM compared with 34 healthy individuals ${ }^{19}$. There is also evidence that GDM is related to chronic insulin resistance, which could subsequently lead to metabolic disturbance ${ }^{20}$. In addition, variations in plasma cytokine levels and oxidative stress biomarkers have been documented among women with a history of $\mathrm{GDM}^{21-23}$. Di Cianni et al. ${ }^{22}$ observed that the prevalence of metabolic syndrome and c-reactive protein levels at 16 months after delivery are significantly higher in individuals with a previous history of GDM; inflammatory markers have been shown to predict future cardiovascular events ${ }^{24}$.

Our study findings have important clinical implications for the prevention of CVD among women with prior GDM. The public health burden of T2DM attributable to GDM is significant, with up to $31 \%$ of parous women who are diagnosed with T2DM having a history of $\mathrm{GDM}^{25}$. Moreover, a substantial proportion of women with previous GDM progress into abnormal glucose tolerance and T2DM ${ }^{1,26,27}$. The American Diabetes Association (ADA) guideline recommends that women with prior GDM or overt T2DM during pregnancy should undergo a $75 \mathrm{~g}$ oral glucose tolerance test (OGTT) for diabetes during 6 to 12 weeks postpartum and at least every 3 years thereafter ${ }^{28}$. Nevertheless, adherence to the recommendation is very low ${ }^{29-32}$. It is required to develop strategies to increase the rates of follow-up testing among the high-risk population. Moreover, lifestyle interventions targeting nutrition and physical activity have been shown to be effective in mitigating the progression to T2DM in individuals at risk for the disease, including women with a known history of GDM ${ }^{33}$. Therefore, in the future, a large well-designed clinical trials or community-based intervention studies are needed to ascertain the benefits of lifestyle or pharmacological interventions in reducing subsequent CVD risks among women with prior GDM.

There are several strengths to our analysis that add to the robustness of our study findings. These include the large sample size and the relatively long duration of follow-up, which allowed a high statistical power to study different types of CVD simultaneously. Furthermore, we adopted a landmark analysis to minimize the influence of guarantee-time bias. The current study also has some noteworthy limitations. Even though our findings were robust and consistent across a range of analyses, we cannot exclude the possibility that the observed associations have been subject to residual confounding, given the observational nature of the present study. In particular, our 


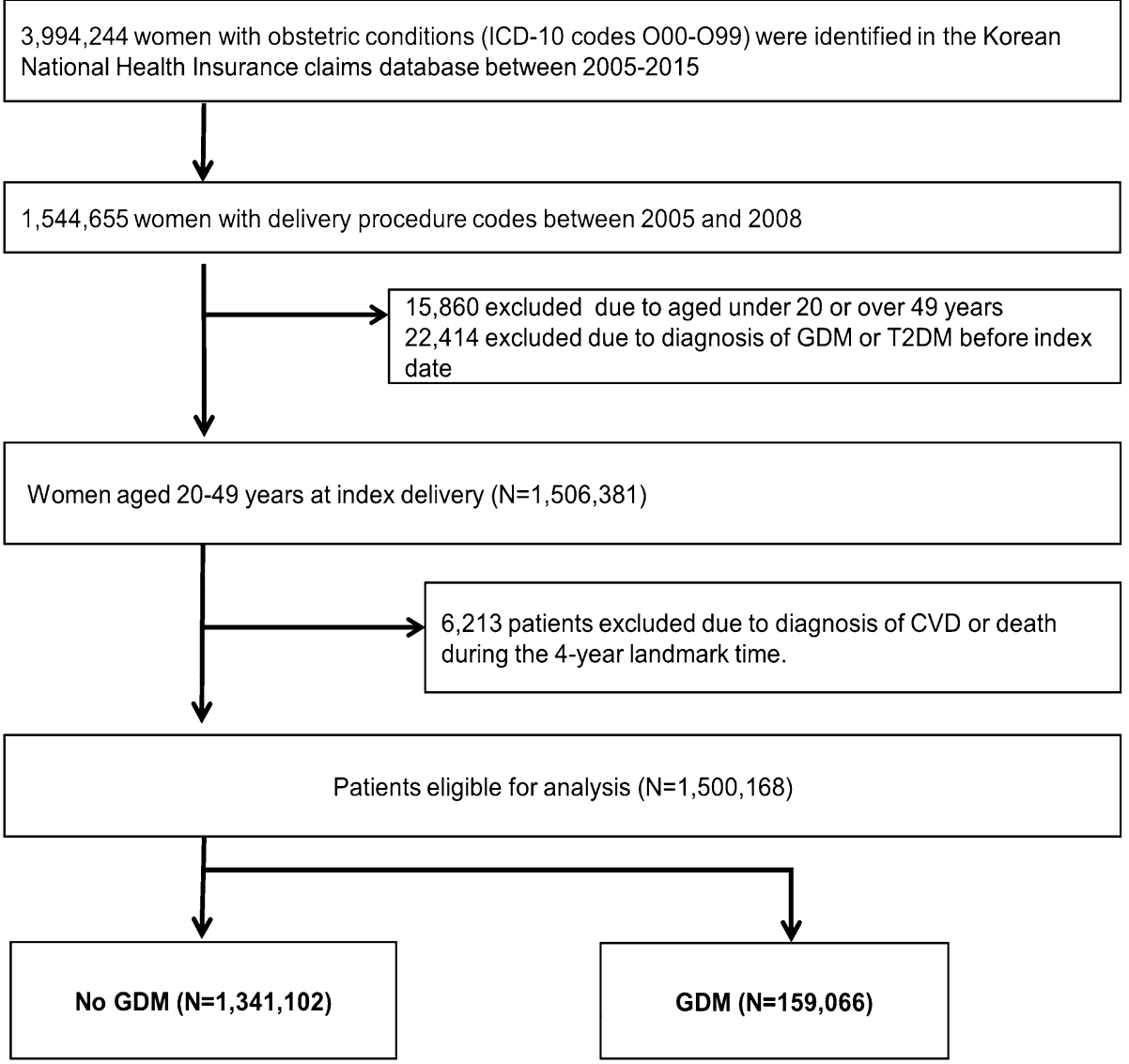

Figure 2. Flow chart of participant selection.

study used administrative data where information on clinical risk factors and other metabolic risk factors, such as gestational weight gain was not available ${ }^{34}$. We were therefore unable to delineate to what extent maternal conditions before or during pregnancy have affected the observed association between GDM and CVD. In addition, death from cardiovascular causes is an important composite outcome of cardiovascular diseases. Lack of such data in the NHIS claims data precluded its inclusion in the present study. Importantly, the claims data do not cover anthropometric and laboratory parameters, such as the oral glucose tolerance test, which is specific to GDM. This made it impossible to determine the severity of GDM. Further study is warranted to evaluate the impacts of severity of GDM on the risk of CVD.

In conclusion, this study provides evidence that women with a history of GDM are at increased risk of CVD, and subsequent progression to T2DM accounts for much of this elevated risk. These findings reinforce the need for implementation of postpartum diabetes interventions to reduce future CVD risk in women with a history of GDM.

\section{Methods}

Data source. This is a retrospective population-based study. The data used came from the 2002-2018 reimbursement claims database of Korea's National Health Insurance Service (NHIS). The data has been described in detail elsewhere ${ }^{35}$. In short, as a single payer, the NHIS provides universal healthcare coverage for all residents in South Korea. The medical providers make claims to the Health Insurance Review and Assessment (HIRA) for reimbursement of the services they provide to patients. This process results in the compilation of a comprehensive source of healthcare utilization data, including demographics, the International Classification of Diseases 10th revision (ICD-10) principal diagnosis codes, procedure codes, prescription records, type of insurance, and medical care costs. This study was approved by the Institutional Review Board (IRB) of Severance Hospital, Yonsei University College of Medicine, Seoul, Korea (IRB no: Y-2018-0121) and the study complied with the Declaration of Helsinki for medical research involving human subjects. The requirement for informed consent was waived as the NHIS database was constructed after anonymization according to strict confidentiality guidelines. 
Study population. From the NHIS claims database, we identified a total of 3,994,244 women with obstetric conditions between 1 January 2005 and 31 December 2015 using the International Classification of Disease 10th revision [ICD-10] codes O00-O99. Among them, 1,544,655 women had given birth between January 1, 2005 and December 31, 2008. Procedure codes were used to identify vaginal and cesarean deliveries and the date of the first claim indicating delivery was used as the index delivery. For each participant, index date (date of conception) was calculated by subtracting 266 days ( 38 weeks) from the date of index delivery ${ }^{36,37}$. Our analysis was restricted to women aged between 20 to 49 years. To ensure that incidence risk, based on the period from the first GDM or T2DM occurrence to cardiovascular disease (CVD), could be accurately estimated, prevalent cases with GDM and T2DM before the index were excluded from our study. Additionally, participants with death, or CVD diagnosis within 4 years from the index date were also excluded. Consequently, the final study cohort consisted of 1,500,168 women. The enrollment flowchart for this study is illustrated in Fig. 2.

Exposure assessment. In Korea, all pregnant women between 24 and 28 weeks of pregnancy are screened for GDM. The one-step method or the two-step method are both used for diagnosing GDM ${ }^{38}$. In the two-step method, pregnancy women then undergo a 100-g, 3-h oral glucose tolerance test (OGTT) after the non-fasting 50 -g challenge test. The threshold values based on the criteria of Carpenter and Coustan with two abnormal values required for diagnosing $\mathrm{GDM}^{39}$. Since data on the OGTT test are not available in the NHIS claims data, International Classification of Diseases (ICD) 10th revision codes O24.4 and O24.9 during pregnancy were used to identify GDM cases. We classified patients as having GDM if they were diagnosed by the predetermined time point (within 4 years from the index date) and as unexposed otherwise, regardless of subsequent changes in exposure status. In further analyses, we accounted for intercurrent T2DM by categorizing the exposure status into 4 groups: without GDM or T2DM, GDM only, T2DM only, or both GDM and T2DM. Subjects without GDM or T2DM were set as the reference group. Patients were classified as having T2DM if they were screened negative during pregnancy followed by at least one claim with a diagnosis of T2DM (ICD-10: E11), either in outpatient or inpatient care, and were prescribed at least one of the following antidiabetic drug: dipeptidyl peptidase-4 inhibitors, $\alpha$-glucosidase inhibitors, biguanides, meglitinides, sulfonylureas, thiazolidinedione, and insulin.

Ascertainment of CVD outcomes. The primary outcome of interest was the first occurrence of any CVD, defined as hospitalization with a primary diagnosis of myocardial infarction (ICD-10: I21-23), treatment with coronary revascularization (Procedure code: M6651-2, M6561, M6563-4, M6571-2, O1641-2, O1647, OA641-2, OA647), heart failure (ICD-10: I50), and cerebrovascular disease (ICD-10: I60-69).

Covariates. Potential confounders were selected based on a priori assumptions of their relationships with both GDM and CVD ${ }^{40-42}$. Confounders included maternal age at index date, parity number, household income, polycystic ovarian syndrome, history of preeclampsia or hypertension and history of dyslipidemia. Maternal age at index delivery was categorized as $20-24,25-29,30-34,35-39,>40$ years. Total parity was assessed during the landmark time and was categorized as 1,2 , or $\geq 3$. Average monthly insurance premium at year of the index date was used as proxy of household income and was categorized into four groups based on quartiles (Low, middle-low, middle-high, or high). Polycystic ovarian syndrome (PCOS) was identified based on the diagnosis of PCOS (ICD-10: E28.2). History of preeclampsia or hypertension and history of dyslipidemia was confirmed based on the presence of diagnostic codes (Preeclampsia or hypertension ICD-10: O13-16, I10-15, dyslipidemia ICD-10: E78).

Statistical analysis. The data were presented as frequencies and percentages. The differences in patient characteristics between groups of GDM and non-GDM were compared using the Chi-square test. Incidence rates were expressed as the number of new cases of CVD per 100,000 person-years of follow-up. In this study, Cox proportional hazard models were used to assess the association between GDM and incident CVD events, using landmark analysis at 4-year time point. Specifically, in order to ensure that every participant had equal exposure window for GDM exposure and to avoid guarantee-time bias, we selected a fixed time point, known as the landmark time, and survival analysis was conducted on only those subjects who have remained event-free at the specified time period (4 years from the index date) ${ }^{43,44}$. If guarantee-time bias is not accounted for in the analysis, biased estimates of the exposure effects can be caused, in favor of the exposure group ${ }^{45,46}$. All participants were followed up from the landmark point until the outcome of interest, death, or the end of study (31 December, 2018), whichever occurred first. Women without the event of interest by the end of the study were censored. In addition, we used an exposure window of 2 years in a sensitivity analysis to evaluate whether the arbitrarily chosen landmark time would affect the study results. Furthermore, cumulative distribution functions for CVD were estimated using the Kaplan-Meier method and the log-rank test was used to compare incidence of CVD events between the exposure groups. Two-sided P values less than 0.05 were considered statistically significant. Statistical analyses were performed using the SAS ver. 9.4 software and R 3.5.2 (R Foundation for Statistical Computing, Vienna, Austria). Post-hoc power estimation was performed using PASS 2019 software. SAS and all other SAS Institute Inc. product or service names are registered trademarks or trademarks of SAS Institute Inc. in the USA and other countries.

\section{Data availability}

All data generated or analysed during this study are not publicly available due to restrictions by the Korean NHIS. However, interested parties may submit applications to NHIS for access (http://nhiss.nhis.or.kr). 
Received: 15 February 2021; Accepted: 1 October 2021

Published online: 27 October 2021

\section{References}

1. Kim, C., Newton, K. M. \& Knopp, R. H. Gestational diabetes and the incidence of type 2 diabetes: A systematic review. Diabetes Care 25, 1862-1868 (2002).

2. American Diabetes Association. Diagnosis and classification of diabetes mellitus. Diabetes Care 28, S5-S10 (2005).

3. Fadl, H. et al. Gestational diabetes mellitus and later cardiovascular disease: A Swedish population based case-control study. BJOG 121, 1530-1536 (2014).

4. Bellamy, L., Casas, J.-P., Hingorani, A. D. \& Williams, D. J. T. L. Type 2 diabetes mellitus after gestational diabetes: A systematic review and meta-analysis. Lancet 373, 1773-1779 (2009).

5. Carr, D. B. et al. Gestational diabetes mellitus increases the risk of cardiovascular disease in women with a family history of type 2 diabetes. Diabetes Care 29, 2078-2083 (2006).

6. Shah, B. R., Retnakaran, R. \& Booth, G. L. Increased risk of cardiovascular disease in young women following gestational diabetes mellitus. Diabetes Care 31, 1668-1669 (2008).

7. Tobias, D. K. et al. Association of history of gestational diabetes with long-term cardiovascular disease risk in a large prospective cohort of US women. JAMA Intern. Med. 177, 1735-1742 (2017).

8. Goueslard, K. et al. Early cardiovascular events in women with a history of gestational diabetes mellitus. Cardiovasc. Diabetol. 15, $1-7$ (2016).

9. Roth, G. A. et al. Global burden of cardiovascular diseases and risk factors, 1990-2019: Update from the GBD 2019 study. J. Am. Coll. Cardiol. 76, 2982-3021 (2020).

10. S. Mendis, P. Puska, B. Norrving Global Atlas on Cardiovascular Disease Prevention and Control: World Health Organization (2011)

11. World Heart Federation. Women and cardiovascular disease. (2013).

12. Hsieh, F. \& Lavori, P. W. Sample-size calculations for the Cox proportional hazards regression model with nonbinary covariates. Control. Clin. Trials 21, 552-560 (2000).

13. Schoenfeld, D. A. Sample-size formula for the proportional-hazards regression model. Biometrics 39, 499-503 (1983).

14. Kramer, C. K., Campbell, S. \& Retnakaran, R. Gestational diabetes and the risk of cardiovascular disease in women: A systematic review and meta-analysis. Diabetologia 62, 905-914 (2019).

15. Perales, M. et al. Impact of gestational risk factors on maternal cardiovascular system. Ann. Transl. Med. 4, 253 (2016).

16. Oliveira, A. P. et al. Assessment of structural cardiac abnormalities and diastolic function in women with gestational diabetes mellitus. Diabetes Vasc. Dis. Res. 12, 175-180 (2015).

17. Appiah, D. et al. Association of gestational diabetes mellitus with left ventricular structure and function: The CARDIA study. Diabetes Care 39, 400-407 (2016).

18. Li, S. \& Yang, H. Relationship between advanced glycation end products and gestational diabetes mellitus. J. Matern. Fetal Neonatal Med. 32, 2783-2789 (2019).

19. Zakovicova, E., Charvat, J., Mokra, D., Svab, P. \& Kvapil, M. The optimal control of blood glucose is associated with normal blood pressure 24 hours profile and prevention of the left ventricular remodeling in the patients with gestational diabetes mellitus. Neuroendocrinol. Lett. 35, 327-333 (2014).

20. Barbour, L. A. et al. Cellular mechanisms for insulin resistance in normal pregnancy and gestational diabetes. Diabetes Care 30, S112-S119 (2007).

21. Heitritter, S. M., Solomon, C. G., Mitchell, G. F., Skali-Ounis, N. \& Seely, E. W. Subclinical inflammation and vascular dysfunction in women with previous gestational diabetes mellitus. J. Clin. Endocrinol. Metab. 90, 3983-3988 (2005).

22. Di Cianni, G. et al. C-reactive protein and metabolic syndrome in women with previous gestational diabetes. Diabetes Metab. Res. Rev. 23, 135-140 (2007).

23. Gomes, C. P. et al. Cytokine levels in gestational diabetes mellitus: A systematic review of the literature. Am. J. Reprod. Immunol. 69, 545-557 (2013).

24. Di Cianni, G. et al. C-reactive protein and metabolic syndrome in women with previous gestational diabetes. J. Diabetes Metab. Res. Rev. 23, 135-140 (2007).

25. Cheung, N. W. \& Byth, K. Population health significance of gestational diabetes. Diabetes Care 26, 2005-2009 (2003).

26. Capula, C. et al. Prevalence and predictors of postpartum glucose intolerance in Italian women with gestational diabetes mellitus. Diabetes Res. Clin. Pract. 105, 223-230 (2014).

27. Benhalima, K., Jegers, K., Devlieger, R., Verhaeghe, J. \& Mathieu, C. Glucose intolerance after a recent history of gestational diabetes based on the 2013 WHO criteria. PLoS One 11, e0157272 (2016).

28. Kim, C., Chames, M. C. \& Johnson, T. R. Identifying post-partum diabetes after gestational diabetes mellitus: The right test. Lancet Diabetes Endocrinol. 1, 84-86 (2013).

29. Kim, C. et al. Missed opportunities for type 2 diabetes mellitus screening among women with a history of gestational diabetes mellitus. Am. J. Public Health 96, 1643-1648 (2006).

30. Cho, G. J. et al. Postpartum glucose testing rates following gestational diabetes mellitus and factors affecting testing non-compliance from four tertiary centers in Korea. J. Korean Med. Sci. 30, 1841-1846 (2015).

31. Kwong, S., Mitchell, R. S., Senior, P. A. \& Chik, C. L. Postpartum diabetes screening: Adherence rate and the performance of fasting plasma glucose versus oral glucose tolerance test. Diabetes Care 32, 2242-2244 (2009).

32. Russell, M. A., Phipps, M. G., Olson, C. L., Welch, H. G. \& Carpenter, M. W. Rates of postpartum glucose testing after gestational diabetes mellitus. Obstet. Gynecol. 108, 1456-1462 (2006).

33. O'Reilly, S. L. et al. Mothers after Gestational Diabetes in Australia (MAGDA): A randomised controlled trial of a postnatal diabetes prevention program. PLoS Med. 13, e1002092 (2016).

34. Sorbye, L., Skjaerven, R., Klungsoyr, K. \& Morken, N.-H. Gestational diabetes mellitus and interpregnancy weight change: A population-based cohort study. PLos Med. 14, e1002367 (2017).

35. Cheol Seong, S. et al. Data resource profile: The national health information database of the National Health Insurance Service in South Korea. Int. J. Epidemiol. 46, 799-800 (2017).

36. Kim, H. O., Lee, K., Lee, S. M. \& Seo, G. H. Association between pregnancy outcomes and radioactive iodine treatment after thyroidectomy among women with thyroid cancer. JAMA Intern. Med. 180, 54-61 (2020).

37. Park, K. Y. et al. Pregnancy outcomes in patients with vitiligo: A nationwide population-based cohort study from Korea. J. Am. Acad. Dermatol. 79, 836-842 (2018).

38. Fuller, K. P. \& Borgida, A. F. Gestational diabetes mellitus screening using the one-step versus two-step method in a high-risk practice. Clin. Diabetes 32, 148-150 (2014).

39. Carpenter, M. W. \& Coustan, D. R. Criteria for screening tests for gestational diabetes. Am. J. Obstet. Gynecol. 144, 768-773 (1982).

40. Parikh, N. I. et al. Parity and risk of later-life maternal cardiovascular disease. Am. Heart J. 159, 215-221.e216 (2010).

41. Lao, T. T., Ho, L.-F., Chan, B. C. \& Leung, W.-C. Maternal age and prevalence of gestational diabetes mellitus. Diabetes Care 29, 948-949 (2006). 
42. Haug, E. B. et al. Association of conventional cardiovascular risk factors with cardiovascular disease after hypertensive disorders of pregnancy: Analysis of the Nord-Trøndelag Health Study. JAMA Cardiol. 4, 628-635 (2019).

43. Dafni, U. Landmark analysis at the 25-year landmark point. Circ. Cardiovasc. Qual. Outcomes 4, 363-371 (2011).

44. Morgan CJ. Landmark analysis: a primer. J Nucl Cardiol. 26(2), 391-393 (2019)

45. Cho, I. S. et al. Statistical methods for elimination of guarantee-time bias in cohort studies: A simulation study. BMC Med. Res. Methodol. 17, 1-7 (2017)

46. Suissa, S. Immortal time bias in pharmacoepidemiology. Am. J. Epidemiol. 167, 492-499 (2008).

\section{Author contributions}

J.S., G.R.K., and S.J.L. were responsible for the conception and design of the study. J.S. and G.R.K. conducted the analyses and drafted the manuscript. H.C.K. revised and commented on the draft, and all authors read and approved the final version of the manuscript.

\section{Funding}

This research was supported by grants from the Korean Society of Cardiovascular Disease Prevention (Grant number 2019-02) and the Graduate School of YONSEI University Research Scholarship Grants in 2017.

\section{Competing interests}

The authors declare no competing interests.

\section{Additional information}

Supplementary Information The online version contains supplementary material available at https://doi.org/ 10.1038/s41598-021-99993-4.

Correspondence and requests for materials should be addressed to S.J.L. or H.C.K.

Reprints and permissions information is available at www.nature.com/reprints.

Publisher's note Springer Nature remains neutral with regard to jurisdictional claims in published maps and institutional affiliations.

(c) (i) Open Access This article is licensed under a Creative Commons Attribution 4.0 International License, which permits use, sharing, adaptation, distribution and reproduction in any medium or format, as long as you give appropriate credit to the original author(s) and the source, provide a link to the Creative Commons licence, and indicate if changes were made. The images or other third party material in this article are included in the article's Creative Commons licence, unless indicated otherwise in a credit line to the material. If material is not included in the article's Creative Commons licence and your intended use is not permitted by statutory regulation or exceeds the permitted use, you will need to obtain permission directly from the copyright holder. To view a copy of this licence, visit http://creativecommons.org/licenses/by/4.0/.

(C) The Author(s) 2021 